\title{
Prevalence and predictors of posttraumatic stress disorder, depression and anxiety among hospitalized patients with coronavirus disease 2019 in China
}

Yaru Chen ${ }^{1,2+}$, Xin Huang ${ }^{1,2+}$, Chengyuan Zhang ${ }^{3}$, Yuanyuan $\mathrm{An}^{4}$, Yiming Liang ${ }^{1,2}$, Yufang Yang ${ }^{1,2}$ and Zhengkui Liu ${ }^{1,2^{*}}$

\begin{abstract}
Background: Coronavirus disease 2019 (COVID-19) has affected more than 5 million people around the world and killed more than 300,000 people; thus, it has become a global public health emergency. Our objective was to investigate the mental health of hospitalized patients diagnosed with COVID-19.

Methods: The PTSD checklist for DSM-5 (PCL-5), Patient Health Questionnaire (PHQ-9), Generalized Anxiety Disorder Scale (GAD-7), Trauma Exposure Scale, abbreviated version of the Connor-Davidson Resilience Scale (CD-RISC-10), Perceived Social Support Scale (PSSS) and Demographic Questionnaire were used to examine posttraumatic stress disorder (PTSD), depression, anxiety, trauma exposure, resilience and perceived social support among 898 patients who were hospitalized after being diagnosed with COVID-19 in China. The data were analyzed with $t$ tests, one-way ANOVA and multivariable logistic regression analysis.

Results: The results showed that the prevalence of PTSD, depression and anxiety was 13.2, 21.0 and 16.4\%, respectively. Hospitalized patients who were more impacted by negative news reports, had greater exposure to traumatic experiences, and had lower levels of perceived social support reported higher PTSD, depression and anxiety.

Conclusions: Effective professional mental health services should be designed to support the psychological wellbeing of hospitalized patients, especially those who have severe disease, are strongly affected by negative news and have high levels of exposure to trauma.
\end{abstract}

Keywords: COVID-19, Hospitalized patients, Posttraumatic stress disorder (PTSD), Depression, Anxiety

\footnotetext{
* Correspondence: liuzk@psych.ac.cn

${ }^{\dagger}$ Yaru Chen and Xin Huang contributed equally to this work.

${ }^{1}$ CAS Key Laboratory of Mental Health, Institute of Psychology, Chinese

Academy of Sciences, 16 Lincui Road, Chaoyang District, Beijing 100101,

China

${ }^{2}$ Department of Psychology, University of Chinese Academy of Sciences, Beijing, China

Full list of author information is available at the end of the article
}

(c) The Author(s). 2021 Open Access This article is licensed under a Creative Commons Attribution 4.0 International License, which permits use, sharing, adaptation, distribution and reproduction in any medium or format, as long as you give appropriate credit to the original author(s) and the source, provide a link to the Creative Commons licence, and indicate if changes were made. The images or other third party material in this article are included in the article's Creative Commons licence, unless indicated otherwise in a credit line to the material. If material is not included in the article's Creative Commons licence and your intended use is not permitted by statutory regulation or exceeds the permitted use, you will need to obtain permission directly from the copyright holder. To view a copy of this licence, visit http://creativecommons.org/licenses/by/4.0/ The Creative Commons Public Domain Dedication waiver (http://creativecommons.org/publicdomain/zero/1.0/) applies to the data made available in this article, unless otherwise stated in a credit line to the data. 


\section{Background}

Coronavirus disease 2019 (COVID-19) has affected more than 5 million people and caused more than 300,000 deaths worldwide in just a few months. The World Health Organization (WHO) declared the outbreak a pandemic in March 2020 and stated that the world was facing a global health crisis [1]. To date, most studies have focused on mental health problems in medical staff and the general population [2-4], and research involving hospitalized patients with COVID-19 has focused on treatments for the disease [5]. As observed during the epidemics of severe acute respiratory syndrome (SARS), novel influenza A (H1N1), Ebola virus disease (EVD) and Middle East respiratory syndrome (MERS), patients experience serious psychological problems in the acute phase of the disease and in the long term after an epidemic [6-10]. Therefore, the mental health problems experienced by hospitalized patients with COVID-19 must be detected and treated promptly. This issue deserves global attention. However, no detailed study on the mental health status of patients during the pandemic has been conducted to date.

According to previous studies, the onset of a sudden and immediately life-threatening illness can lead to posttraumatic stress disorder (PTSD) [11]. The psychological pressure on COVID-19 patients due to isolation treatment and other reasons may be far greater than that of the general public, and the situations they face during hospitalization also produce potential trauma [12]. According to past experiences of epidemics, more than $40 \%$ of SARS survivors experienced posttraumatic stress symptoms at some time during the outbreak [10, 13]. In addition to PTSD, depression and anxiety are common mental health problems in patients with infectious diseases. Kim et al. [7] investigated patients with suspected and confirmed MERS who were isolated in hospitals and found that the prevalence of depression was $40.7 \%$. Wu et al. [14] reported that $14 \%$ of SARS patients reported anxiety 1 month after they were discharged from the hospital. Consequently, PTSD, depression and anxiety in patients during the COVID-19 outbreak should be afforded more attention.

Moreover, PTSD commonly co-occurs with other psychiatric disorders over a range of populations and trauma types [15]. Among subjects with PTSD, the rates of comorbid depression and anxiety range from 21 to $94 \%$ and from 39 to $97 \%$, respectively [16]. Previous studies showed that 10 to $35 \%$ of SARS survivors reported symptoms of depression, anxiety or both during the early recovery phase $[14,17]$. Compared with PTSD alone, PTSD combined with other psychiatric diseases is more difficult to treat [18]. Therefore, it is very important to identify psychiatric comorbidities of PTSD in hospitalized patients with COVID-19 to enable the implementation of interventions as soon as possible. In addition, it is critical to determine the factors that are predictive of these comorbidities.

Evidence has shown that when PTSD and other psychiatric disorders co-occur in populations who have experienced trauma, a combined stress model with shared vulnerabilities and similar risk factors might be involved [18]. Therefore, it is important to identify risk factors for not only the psychiatric disorders of interest but also comorbid disorders. North et al. [19] systematically analyzed the factors predictive of mental health problems in survivors of 10 disasters. Their review noted that the research findings to date collectively indicate that the risk of mental health problems following disasters is generally associated with female sex, young age, minority ethnicity, lower socioeconomic status, higher education level, marital status (married for women and unmarried for men), predisaster psychiatric illness, greater exposure to the disaster, and lack of perceived and actual social support. Focusing on the differences in demographic variables between individuals with relatively high and low scores for PTSD, depression and anxiety helps to predict the populations at high risk for these disorders. This study focuses on sex, age, educational background and socioeconomic status. The degree of trauma exposure is a direct inducer of the posttraumatic psychological response [20]. Trauma exposure refers to objective factors such as the degree of injury to oneself, relatives and friends and subjective experiences such as worrying about the safety of oneself and important others [21]. Studies have found that media exposure during critical public events may cause additional psychological trauma and anxiety, indicating that the vicarious traumatization effect may play an important role in the development of psychological disorders [22, 23]. Hence, exposure to news reports related to the pandemic is considered a candidate risk factor.

In addition, resilience and perceived social support are considered protective factors against an adverse psychological response. Resilience is the ability to maintain relatively stable, healthy levels of psychological and physical functioning in the context of adversity, stress or trauma [24]. After patients have been diagnosed with COVID-19, resilience enables them to cope with the stressful event through the interaction of external resources and internal potential [25]. Perceived social support has been identified as a strong external resource [26]. According to Cohen and Wills [27] and Goyne and Downey [28], there are two models to explain the role of social support in the posttraumatic psychological response. The direct effect model suggests that social support can directly promote individual posttraumatic adaptation by improving an individual's healthy behavior and reducing the negative psychological response after 
the traumatic event. The buffering effect model of social support holds that providing resources for individuals to use when coping with traumatic events helps to alleviate the negative impact of these events on individuals.

This study was performed to provide information about the prevalence rates of PTSD, depression, and anxiety among COVID-19 patients in China and to explore the associated risk factors. The related factors examined were demographic variables, trauma exposure and psychosocial variables.

\section{Methods}

\section{Participants}

Starting in March 2020, we recruited patients who had been hospitalized for COVID-19 at the end of January and early February in China, primarily from Wuhan in Hubei Province. The patients began to return to the hospital for reexamination in the middle of March.

A total of 909 hospitalized patients with COVID-19 were recruited as of May 2020. After eliminating 5 participants aged under 16 years old, 1 participant who reported a history of psychiatric disease and 5 participants who were invalidated due to incomplete or careless answers, the final number of participants was 898 . The sociodemographic information is shown in Table 1 . The mean age of the participants was 39.40 years $(S D=$ 14.05), with a range from 16 to 92 years. Of the participants, $123(13.7 \%)$ were younger than 20 years old, $336(37.4 \%)$ were between 21 and 40, 370 (41.2\%) were between 41 and 60, and $62(6.9 \%)$ were over 60 years. A total of $382(42.5 \%)$ participants were male. In terms of educational background, 127 (14.1\%) participants had a diploma from a primary or secondary school, 204 (22.7\%) participants had a diploma from high school or technical secondary school, 532 (59.2\%) participants had a diploma from a university or technical college, and 35 (3.9\%) participants had a graduate degree or above. In terms of subjective socioeconomic status, 96 (10.7\%) participants were at a low level, 316 (35.2\%) participants were at a below-average level, $449(50.0 \%)$ participants were at an average level, and 37 (4.1\%) participants were at an above-average level or high level.

\section{Procedures}

From March 22, 2020, to May 24, 2020, medical and psychological health care workers in hospitals for COVID-19 introduced this investigation to patients with COVID-19. Due to the pandemic disease prevention and control measures, the questionnaire was completed online in the WeChat application after informed consent was obtained. The method of completing the questionnaire was very simple. The participants clicked the link shared by the psychological health care workers to access the questionnaire. After answering all the questions,

Table 1 Demographic differences among patients with PTSD, depression and anxiety $(n=898)$

\begin{tabular}{|c|c|c|c|c|c|c|c|c|c|c|c|c|c|c|}
\hline & \multicolumn{3}{|c|}{ Respondents } & \multicolumn{4}{|l|}{ PTSD } & \multicolumn{4}{|c|}{ Depression } & \multicolumn{3}{|c|}{ Anxiety } \\
\hline & $\mathrm{N}$ & $\%$ & $M$ & $S D$ & $F / t$ & $p$-value & $M$ & $S D$ & $F / t$ & $p$-value & $M$ & $S D$ & $F / t$ & $p$-value \\
\hline Overall & 898 & 100 & 15.88 & 14.62 & & & 6.72 & 5.62 & & & 4.71 & 5.12 & & \\
\hline \multicolumn{15}{|l|}{ Sex } \\
\hline Male & 382 & 42.5 & 15.06 & 14.46 & -1.44 & .152 & 5.91 & 5.53 & -1.66 & .097 & 4.31 & 5.01 & -2.04 & .041 \\
\hline Female & 516 & 57.5 & 16.48 & 14.72 & & & 6.54 & 5.67 & & & 5.01 & 5.19 & & \\
\hline \multicolumn{15}{|l|}{ Age, years } \\
\hline$\leq 20$ & 123 & 13.7 & 11.81 & 14.50 & 4.84 & .002 & 5.54 & 5.19 & 0.91 & .435 & 3.06 & 4.58 & 5.34 & .001 \\
\hline $21-40$ & 336 & 37.4 & 15.64 & 15.40 & & & 6.45 & 5.95 & & & 5.01 & 5.40 & & \\
\hline $41-60$ & 370 & 41.2 & 16.98 & 13.84 & & & 6.28 & 5.44 & & & 4.89 & 4.94 & & \\
\hline$\geq 61$ & 62 & 6.9 & 18.90 & 14.62 & & & 6.69 & 5.62 & & & 5.50 & 5.24 & & \\
\hline \multicolumn{15}{|l|}{ Educational background } \\
\hline Primary and secondary school & 127 & 14.1 & 19.49 & 15.58 & 4.17 & .006 & 6.64 & 5.73 & 0.33 & .801 & 5.63 & 5.45 & 3.63 & .013 \\
\hline High school or technical school & 204 & 22.7 & 16.75 & 13.33 & & & 6.27 & 5.32 & & & 5.15 & 4.90 & & \\
\hline University or college & 532 & 59.2 & 14.64 & 14.69 & & & 6.15 & 5.70 & & & 4.26 & 5.01 & & \\
\hline Postgraduate or above & 35 & 3.9 & 16.54 & 15.11 & & & 6.71 & 5.85 & & & 5.66 & 6.25 & & \\
\hline \multicolumn{15}{|c|}{ Socioeconomic status } \\
\hline Low & 96 & 10.7 & 19.51 & 16.99 & 3.32 & .019 & 7.45 & 6.47 & 3.62 & .013 & 6.27 & 5.93 & 4.45 & .004 \\
\hline Below-average & 316 & 35.2 & 16.58 & 14.76 & & & 6.68 & 5.83 & & & 4.87 & 5.20 & & \\
\hline Average & 449 & 50.0 & 14.66 & 13.84 & & & 5.69 & 5.21 & & & 4.24 & 4.77 & & \\
\hline Above-average to high & 37 & 4.1 & 15.14 & 14.60 & & & 6.62 & 5.73 & & & 5.08 & 5.62 & & \\
\hline
\end{tabular}


the patients then clicked the submit button. If the participants had any operational questions or difficulties completing the questionnaire, the psychological health care workers provided guidance. The patients were notified that online and in-person psychological counseling services were available if needed after the survey.

The study design and procedures were approved by the ethics review committee of the Institute of Psychology, Chinese Academy of Sciences (Protocol name: Evaluation and intervention of mental health of people affected by COVID-19).

\section{Measures}

\section{PTSD checklist for DSM-5 (PCL-5)}

PTSD was assessed with the PTSD checklist for DSM-5 (PCL-5), which was compiled by Weathers and colleagues [29], translated into Chinese and revised by Zhou, Wu and Zhen [30]. A total of 20 items rated on a 5 -point scale ranging from 0 (never) to 4 (severe) were used to assess the frequency of symptoms after diagnosis with COVID-19. The participants were asked to report their symptoms in the last month. The PCL-5 is composed of four dimensions: intrusion, emotion alteration, avoidance, and hyperarousal. A total score is computed for each item, with higher scores indicating a higher degree of PTSD symptoms. Based on the clinical criteria [31], scores above 33 indicate probable posttraumatic stress symptoms. In the current study, Cronbach's alpha for the scale was 0.962 .

\section{Patient health questionnaire (PHQ-9)}

Depression was assessed with the Patient Health Questionnaire (PHQ-9), which was compiled by Kroenke, Spitzer, and Williams [32] and translated into Chinese and revised by Wang et al. [33]. A total of 9 items rated on a 4-point scale ranging from 0 (not at all) to 3 (almost every day) were used to assess the frequency of symptoms in the past 2 weeks. Based on the established criteria [34], scores of 10-14 indicate moderate depression, scores of 15-19 indicate severe depression, and scores of 20-27 indicate severe depression. In the current study, Cronbach's alpha for the scale was 0.920 .

\section{Generalized anxiety disorder scale (GAD-7)}

Anxiety was assessed with the Chinese version of the Generalized Anxiety Disorder Scale (GAD-7) [35]. A total of 7 items rated on a 4-point scale ranging from 0 (never) to 3 (almost every day) were used to assess the frequency of symptoms in the past 2 weeks. According to the established criteria [36], scores of 10-14 indicate moderate anxiety and scores of 15-21 indicate severe anxiety. In the current study, Cronbach's alpha for the scale was 0.954 .

\section{Trauma exposure scale}

Trauma exposure was assessed with the second part of the University of California at Los Angeles (UCLA) PTSD reaction index (UCLA PTSD-RI) [37]. A total of 13 items were used to assess the objective (e.g., Were you seriously ill?) and subjective (e.g., Did you feel very scared, like this was one of your scariest experiences ever?) features of exposure to trauma. These items were scored as present (1) or absent (0). A total score for the 13 items was used to indicate the degree of trauma exposure in the current study.

\section{Abbreviated version of the Connor-Davidson resilience scale (CD-RISC-10)}

Resilience was assessed with the abbreviated version of the Connor-Davidson Resilience Scale (CD-RISC-10), which was compiled by Connor and Davidson [38] and revised by Wang et al. [39]. A total of 10 items rated on a 5-point scale ranging from 0 (not true at all) to 4 (true nearly all the time) were used to assess agreement with statements of psychological regulation in the last month. Higher total scores indicate a high level of resilience. In the current study, Cronbach's alpha for the scale was 0.969 .

\section{Perceived social support scale (PSSS)}

Perceived social support was assessed with the Chinese version of the Perceived Social Support Scale (PSSS) [40]. A total of 12 items were rated on a 7-point scale ranging from 1 (extremely disagree) to 7 (extremely agree). The scale is composed of two dimensions: family endogenous support and family exogenous support. A total score is computed for each dimension, with higher scores indicating a higher level of perceived social support. In the current study, Cronbach's alpha for the full scale was 0.954 .

\section{Sociodemographic information}

Participants were asked to report their sex, age, educational background, socioeconomic status and the impact of news reports. One item was used to assess subjective socioeconomic status (e.g., How do you think the living standard of your family compares to that of the whole country?) ranging from 1 (low) to 4 (above-average to high). Two items were used to assess the negative impact of news reports (e.g., To what extent have you been negatively affected by news coverage of COVID-19?) and the positive impact of news reports (e.g., To what extent have you been positively affected by news coverage of COVID-19?) ranging from 1 (almost none) to 6 (almost all). 


\section{Data analysis}

Data were analyzed with SPSS 26.0. Independent sample $t$ tests and one-way ANOVA were used to examine differences in sociodemographic information among patients with different scores for PTSD, depression and anxiety. Multivariate logistic regression analyses were performed to identify the risk factors for PTSD, depression and anxiety. All statistically significant associated variables in Table 1 were collectively entered into multivariable logistic regression analyses. Odds ratios (ORs) with $95 \%$ confidence intervals (95\% CIs) were calculated to measure the strength of association. All statistical tests were 2 -tailed, and a $p$-value of less than .05 considered statistically significant.

\section{Results}

Prevalence of PTSD, depression and anxiety and the associated demographics

Using a cutoff score of 33, 119 (13.2\%) patients had probable posttraumatic stress symptoms. Using a cutoff score of 10 , the prevalence of depression was $21.0 \%$, and the prevalence of moderate, severe and very severe depression was 11.5, 6.3 and 3.2\%, respectively. Using a cutoff score of 10 , the prevalence of anxiety was $16.4 \%$, and the prevalence of moderate and severe anxiety was 10.9 and 5.5\%, respectively. Among the participants with PTSD, 77.3\% had comorbid depression and 65.5\% had comorbid anxiety. Among the participants with depression, the proportion with comorbid anxiety was $65.6 \%$. In total, $8.2 \%$ of the participants had three disorders.

Effect of sociodemographics, trauma exposure, resilience and perceived social support on PTSD, depression and anxiety

The results of the multivariable logistic regression analysis are presented in Table 2. Hospitalized patients who were more impacted by negative news reports $(\mathrm{OR}=$ $1.72,95 \% \mathrm{CI}=1.37 \sim 2.15$ for PTSD; $\mathrm{OR}=1.69,95 \% \mathrm{CI}=$ $1.40 \sim 2.05$ for depression; $\mathrm{OR}=1.62,95 \% \mathrm{CI}=1.32 \sim$ $1.99)$, had greater exposure to traumatic experiences $(\mathrm{OR}=1.20,95 \% \mathrm{CI}=1.12 \sim 1.29$ for PTSD; OR $=1.25$, $95 \% \mathrm{CI}=1.18 \sim 1.33$ for depression; $\mathrm{OR}=1.24,95 \% \mathrm{CI}=$ $1.15 \sim 1.32$ ), and had lower levels of perceived social support $(\mathrm{OR}=0.96,95 \% \mathrm{CI}=0.95 \sim 1.00$ for PTSD; OR =

Table 2 Multivariable logistic regression analyses of possible factors associated with PTSD, depression and anxiety $(n=898)$

\begin{tabular}{|c|c|c|c|c|c|c|}
\hline & \multicolumn{2}{|l|}{ PTSD } & \multicolumn{2}{|l|}{ Depression } & \multicolumn{2}{|l|}{ Anxiety } \\
\hline & OR $(95 \% \mathrm{CI})$ & $p$-value & OR $(95 \% \mathrm{CI})$ & $p$-value & OR $(95 \% \mathrm{Cl})$ & $p$-value \\
\hline \multicolumn{7}{|l|}{ Sex } \\
\hline Male & $1.04(0.66,1.61)$ & .881 & $1.04(0.71,1.52)$ & & $1.01(0.67,1.53)$ & .955 \\
\hline Female & 1 & & 1 & & 1 & \\
\hline \multicolumn{7}{|l|}{ Age, years } \\
\hline$\leq 20$ & $1.06(0.37,3.03)$ & .912 & $1.57(0.61,4.05)$ & .355 & $0.65(0.23,1.81)$ & .405 \\
\hline $21-40$ & $0.79(0.33,1.88)$ & .592 & $1.31(0.58,2.93)$ & .516 & $0.86(0.39,1.93)$ & .720 \\
\hline $41-60$ & $0.57(0.25,1.30)$ & .182 & $0.96(0.45,2.07)$ & .915 & $0.56(0.26,1.20)$ & .134 \\
\hline$\geq 61$ & 1 & & 1 & & & \\
\hline \multicolumn{7}{|l|}{ Educational background } \\
\hline Primary and secondary school & $0.95(0.28,3.31)$ & .941 & $0.74(0.26,2.12)$ & .573 & $0.49(0.17,1.45)$ & .199 \\
\hline High school or technical school & $0.81(0.25,2.59)$ & .721 & $0.73(0.27,1.92)$ & .519 & $0.56(0.21,1.79)$ & .249 \\
\hline University or college & $1.02(0.34,3.03)$ & .972 & $0.94(0.38,2.35)$ & .902 & $0.57(0.22,1.51)$ & .232 \\
\hline Postgraduate or above & 1 & & 1 & & 1 & \\
\hline \multicolumn{7}{|l|}{ Socioeconomic status } \\
\hline Low & $1.96(0.53,7.31)$ & .314 & $0.93(0.33,2.61)$ & .893 & $1.23(0.42,3.58)$ & .711 \\
\hline Below-average & $1.52(0.46,5.18)$ & .505 & $0.72(0.28,1.83)$ & .491 & $0.67(0.25,1.79)$ & .425 \\
\hline Average & $1.31(0.39,4.43)$ & .666 & $0.54(0.21,1.34)$ & .181 & $0.57(0.22,1.51)$ & .262 \\
\hline Above-average to high & 1 & & 1 & & 1 & \\
\hline Impact of negative news reports & $1.72(1.37,2.15)$ & $<.001$ & $1.69(1.40,2.05)$ & $<.001$ & $1.62(1.32,1.99)$ & $<.001$ \\
\hline Impact of positive news reports & $1.07(0.86,1.33)$ & .552 & $0.90(0.74,1.09)$ & .270 & $1.05(0.86,1.29)$ & .635 \\
\hline Trauma exposure & $1.20(1.12,1.29)$ & $<.001$ & $1.25(1.18,1.33)$ & $<.001$ & $1.24(1.15,1.32)$ & $<.001$ \\
\hline Resilience & $0.98(0.95,1.00)$ & .080 & $0.98(0.96,1.00)$ & .030 & $0.99(0.96,1.01)$ & .220 \\
\hline Perceived social support & $0.96(0.95,0.98)$ & $<.001$ & $0.97(0.96,0.98)$ & $<.001$ & $0.97(0.95,0.98)$ & $<.001$ \\
\hline
\end{tabular}


0.97, $95 \% \mathrm{CI}=0.96 \sim 0.98$ for depression; $\mathrm{OR}=0.97$, $95 \% \mathrm{CI}=0.95 \sim 0.98)$ were at a higher risk of PTSD, depression and anxiety. Hospitalized patients who had lower levels of resilience $(\mathrm{OR}=0.98,95 \% \mathrm{CI}=0.96 \sim$ 1.00) were at a higher risk of depression.

\section{Discussion}

To the best of our knowledge, this is the first study to investigate the prevalence and predictors of psychiatric symptoms among hospitalized COVID-19 patients during the acute treatment period. The current study showed that the prevalence rates of PTSD, depression and anxiety among hospitalized patients with COVID-19 were high and that these disorders often co-occurred. Negative media reports, exposure to trauma and perceived social support were shared risk and protective factors of PTSD, anxiety and depression. Resilience was a protective factor only for depression.

\section{Prevalence of and differences in PTSD, depression and anxiety among hospitalized patients with COVID-19}

In this study, the prevalence of PTSD, depression and anxiety among hospitalized patients with COVID-19 was 13.2, 21.0 and $16.4 \%$, respectively. The prevalence of PTSD was lower than the prevalence in previous studies with patients with similar infectious diseases $[10,13]$ but higher than those in other groups, such as general residents and medical staff, during the COVID-19 pandemic [2-4]. The prevalence of depression and anxiety was higher than in previous studies on infectious diseases [7, 14] and studies involving residents [4] and medical workers [2] in Wuhan during the COVID-19 outbreak. The results indicate that hospitalized patients who have contracted COVID-19 may have the most serious mental health problems during this pandemic. The possible causes are related to the virus itself, the use of corticosteroids or hydroxychloroquine, or pandemic-related stress. First, the virus that causes COVID-19 might infect the brain or trigger immune responses that have additional adverse effects on brain function and mental health in patients with COVID-19 [41, 42]. Second, corticosteroids may induce affective psychosis, and hydroxychloroquine use has been related to agitation, emotional lability and irritability $[43,44]$. Third, the psychological pressure on COVID-19 patients due to isolation treatment may be far greater than that of medical workers and general residents [12]. Additionally, patients have a greater risk of death.

The study showed that PTSD, depression and anxiety are often comorbid. PTSD, depression, and generalized anxiety disorder (GAD) are all thought to stem from high levels of general distress in the acute stage of trauma and are likely to become a symptom network [45]. A network analysis study showed that symptoms of
GAD (inability to relax) and PTSD (restricted or diminished positive emotion) were identified as key hub symptoms for the network of PTSD, depression and anxiety. Symptoms of depression and GAD are highly interrelated [46]. A previous study has shown that the association of anxiety and depression during the COVID-19 pandemic can be attributed to the strong connection among impaired motor skills, restlessness, and inability to relax [47]. During treatment for COVID-19, patients may develop multiple related psychiatric diseases that form a mutually influential symptom network. Therefore, mental illness intervention from the perspective of symptoms (e.g., impaired motor skills or inability to relax) rather than mental illness as a whole (e.g., PTSD or depression) may be a better choice for mental health interventions for patients. In particular, the relationship between symptoms in the context of COVID-19 needs to be studied further.

\section{Effects of trauma exposure, resilience and perceived social support on PTSD, depression and anxiety}

This study found that negative media reports, trauma exposure and perceived social support were shared risk and protective factors of PTSD, anxiety and depression. Resilience was a protective factor only for depression. First, infection with an unknown virus for which there are no known treatments increases patients' perception of a threat to their lives, which in turn can lead to the development of mental disorders. Second, as one aspect of trauma exposure, the degree of subjective fear plays an important role in inpatients' susceptibility to mental health problems. Previous studies have reported that the subjective experience of fear is more strongly related to PTSD than objective exposure alone [48]. As evidenced in past epidemics [49], the perception of personal risk, rather than not only actual exposure, can confer considerable susceptibility to mental health disorders. Negative news reports and the severity of the disease can affect an individual's subjective experience of fear. Sensational media reports may stimulate the subconscious perception of threat and induce fear [50], and fear has been shown to be associated with a considerable risk of the new onset and recurrence of mental health disorders [51]. Moreover, heightened distress responses to media reports of collective crises may have long-term physical health repercussions [22]. However, the positive impacts of media reports were not found to alleviate psychiatric disorders in this study.

In addition, resilience was a protective factor against depression, and perceived social support was a protective factor against PTSD, depression and anxiety. A possible reason is that external resources are more important than internal resources for patients infected with COVID-19. Social support can not only affect 
individuals directly by improving their health behaviors but can also affect individuals indirectly by providing resources to individuals $[27,28]$. Resilience is a personality trait. In the face of life-threatening events such as COVID-19 infection, patients need to deal with their physical health rather than their mental health. However, according to previous studies, resilience plays an important role in the long-term mental health recovery of patients [11].

\section{Limitations and implications}

There are some limitations of this study. First, our participants were a convenience sample mainly recruited from hospitals in Wuhan. It is uncertain whether our findings can be generalized to all patients during this pandemic. Second, the data used in this study came from patients' self-reported questionnaires. In the future, clinical diagnoses of PTSD, depression and anxiety should be used. Third, this was a cross-sectional study. Directionality cannot be determined between the "predictors" (e.g., resilience, perceived social support) and mental health symptoms. The pattern and long-term clinical course of the psychiatric effects of COVID-19 should continue to be investigated, and there should be widespread awareness of the possible psychiatric impacts of a future reemergence of COVID-19. Future studies should exploit existing datasets and ongoing longitudinal studies in addition to establishing new cohorts to collect detailed information on psychological factors.

The findings of this study indicate that timely integrated mental health interventions are warranted for patients during the acute treatment phase of COVID-19. First, health authorities need to identify high-risk groups based on sociodemographic information and implement early psychological interventions. Females, elderly patients, patients with lower levels of education, and patients with lower socioeconomic status are target populations. Second, reducing news reports during the pandemic and disseminating accurate scientific information will help patients understand this novel virus, thereby alleviating their fear and reducing their perception of COVID-19 as a threat. Third, providing a supportive hospitalization environment will help foster resilience, enabling hospitalized patients to maintain relatively stable, healthy levels of psychological and physical functioning.

\section{Conclusions}

The current study showed that the prevalence rates of PTSD, depression and anxiety among hospitalized patients with COVID-19 were high and that these disorders often co-occurred. Negative media reports, exposure to trauma and perceived social support were shared predictors of PTSD, anxiety and depression.
Resilience was a protective factor only for depression. These findings indicate that timely integrated mental health interventions are warranted for patients during the acute treatment phase of COVID-19.

\section{Acknowledgements \\ Not applicable.}

\section{Authors' contributions}

Yaru Chen analyzed the data and was a major contributor in writing the manuscript. Xin Huang and Chengyuan Zhang collected the data. Xinhuang, Yuanyuan An, Yiming Liang and Zhengkui Liu substantively revised it. Yufang Yang and Zhengkui Liu provided foundations. All authors read and approved the final manuscript.

\section{Funding}

This work was supported by the National Key R \& D Program of China [grant numbers 2020YFC2003000] and the Baidu Research Foundation.

\section{Availability of data and materials}

The datasets used and analyzed during the current study are available from the corresponding author on reasonable request.

\section{Ethics approval and consent to participate}

This study was conducted in accordance with the ethical standards put forth in the Declaration of Helsinki. The questionnaire was completed online in the WeChat application after informed consent was obtained. All subjects under the age of 18 who participated in this study did so after informed consent had been obtained from their parents. The study design and procedures were approved by the ethics review committee of the Institute of Psychology, Chinese Academy of Sciences (Protocol name: Evaluation and intervention of mental health of people affected by COVID-19).

\section{Consent for publication}

Not applicable.

\section{Competing interests}

The authors declare that they have no competing interests.

\section{Author details}

${ }^{1}$ CAS Key Laboratory of Mental Health, Institute of Psychology, Chinese Academy of Sciences, 16 Lincui Road, Chaoyang District, Beijing 100101, China. ${ }^{2}$ Department of Psychology, University of Chinese Academy of Sciences, Beijing, China. ${ }^{3}$ Department of Respiratory and Critical Care Medicine, East District of the First Affiliated Hospital of Anhui Medical University (Feidong People's Hospital), Feidong, China. ${ }^{4}$ School of

Psychology, Nanjing Normal University, Nanjing, China.

Received: 22 October 2020 Accepted: 11 January 2021

Published online: 08 February 2021

\section{References}

1. World Health Organization. COVID-19 related reports. 2020. https://www. who.int/zh/emergencies/diseases/novel-coronavirus-2019. Accessed 1 June 2020.

2. Huang JZ, Han MF, Luo TD, Ren AK, Zhou XP. Mental health survey of 230 medical staff in a tertiary infectious disease hospital for COVID-19. Chin J Industr Hygiene Occup Dis. 2020;38:E001.

3. Liu N, Zhang F, Wei C, Jia Y, Shang Z, Sun L, et al. Prevalence and predictors of PTSS during COVID-19 outbreak in China hardest-hit areas: gender differences matter. Psychiatry Res. 2020;287:112921.

4. Zhao YQ, An YY, Tan X, Li XH. Mental health and its influencing factors among self-isolating ordinary citizens during the beginning epidemic of COVID-19. J Loss Trauma. 2020. https://doi.org/10.1080/15325024.2020. 1761592.

5. Liu C, Zhou QQ, Li YZ, Garner LV, Watkin SP, Carter LJ, et al. Research and Development on therapeutic agents and vaccines for COVID-19 and related human coronavirus diseases. ACS Central Science. 2020;6:315-31. 
6. Jalloh MF, Li W, Bunnell RE, Ethier KA, Redd JT. Impact of ebola experiences and risk perceptions on mental health in Sierra Leone, july 2015. Br Med J Glob Health. 2018;3:e000471.

7. Kim HC, Yoo SY, Lee BH, Lee SH, Shin HS. Psychiatric findings in suspected and confirmed middle east respiratory syndrome patients quarantined in hospital: a retrospective chart analysis. Psychiatry Invest. 2018;15:355.

8. Liao QY, Cowling BJ, Lam WWT, Ng DMW, Fielding R. Anxiety, worry and cognitive risk estimate in relation to protective behaviors during the 2009 influenza a/H1N1 pandemic in Hong Kong: ten cross-sectional surveys. BMC Infect Dis. 2014;14:169.

9. Mak IWC, Chu CM, Pan PC, Yiu MGC, Chan VL. Long-term psychiatric morbidities among sars survivors. Gen Hosp Psychiatry. 2009;31:318-26.

10. Mak IW, Chu CM, Pan PC, Yiu MG, Ho SC, Chan VL. Risk factors for chronic post-traumatic stress disorder (PTSD) in sars survivors. Gen Hosp Psychiatry. 2010;32:590-8.

11. Wu KK, Chan SK, Ma TM. Posttraumatic stress after SARS. Emerg Infect Dis. 2005;11:1297.

12. Xiang YT, Yang Y, Li W, Zhang L, Zhang Q, Cheung T, Ng CH. Timely mental health care for the 2019 novel coronavirus outbreak is urgently needed. Lancet Psychiatry. 2020;7:228-9.

13. Lam MHB, Wing YK, Yu MWM, Leung CM, Ma RCW, Kong APS, et al. Mental morbidities and chronic fatigue in severe acute respiratory syndrome survivors: long-term follow-up. Arch Intern Med. 2009;169:2142-7.

14. Wu KK, Chan SK, Ma TM. Posttraumatic stress, anxiety, and depression in survivors of severe acute respiratory syndrome (SARS). J Traumatic Stress. 2005;18:39-42

15. Angelakis S, Nixon RD. The comorbidity of PTSD and MDD: implications for clinical practice and future research. Behav Chang. 2015;32:1-25.

16. Ginzburg K, Ein-Dor T, Solomon Z. Comorbidity of posttraumatic stress disorder, anxiety and depression: a 20-year longitudinal study of war veterans. J Affect Disord. 2010;123:249-57.

17. Hawryluck L, Gold WL, Robinson S, Pogorski S, Galea S, Styra R. SARS control and psychological effects of quarantine, Toronto, Canada. Emerg Infect Dis. 2004;10:1206.

18. Spinhoven P, Penninx BW, van Hemert AM, de Rooij M, Elzinga BM. Comorbidity of PTSD in anxiety and depressive disorders: prevalence and shared risk factors. Child Abuse Negl. 2014;38:1320-30.

19. North CS, Oliver J, Pandya A. Examining a comprehensive model of disasterrelated posttraumatic stress disorder in systematically studied survivors of 10 disasters. Am J Public Health. 2012;102:e40-8.

20. Fan F, Zhang Y, Yang Y, Mo L, Liu X. Symptoms of postraumatic stres disorder, depresion, and anxiety among adolescents folowing the 2008 Wenchuan earthquake in China. J Trauma Stress. 2011;24:44-53.

21. Wang WC, Wu XC, Zhou X. Prevalence and Predictors of Postraumatic Stres Disorder and Postraumatic Growth among Adolescent Survivors after the Wenchuan Earthquake. J Beijing Normal Univ. 2018;2:51-63.

22. Garfin DR, Silver RC, Holman EA. The novel coronavirus (COVID-2019) outbreak: amplification of public health consequences by media exposure. Health Psychol. 2020;39:355-7.

23. Liu C, Liu Y. Media exposure and anxiety during covid-19: the mediation effect of media vicarious traumatization. International journal of environmental research and public health. 2020. 2020. https://doi.org/10. 3390/ijerph17134720.

24. Bonanno GA. Loss, trauma, and human resilience: have we underestimated the human capacity to thrive after extremely aversive events? Am Psychol. 2004;59:20.

25. Masten AS. Ordinary magic: resilience processes in development. Am Psychol. 2001;56:227

26. Bámaca-Colbert MY, Tilghman-Osborne EM, Calderón-López S, Moore AM. Perceptions of support from mothers, fathers, and friends: direct and indirect associations with the psychological adjustment of Mexican-origin girls. J Latina Psychol. 2017:5:142.

27. Cohen S, Wills TA. Stress, social support, and the Bufering hypothesis. Psychol Buletin. 1985;98:310-57.

28. Goyne JC, Downey G. Social factors and psychopathology: stress, social support and the coping processes. Annu Rev Psychol. 1991;42:401-26.

29. Weathers FW, Litz BT, Keane TM, Palmieri PA, Marx BP, Schnurr PP. The ptsd checklist for dsm-5 (pcl-5). Scale available from the National Center for PTSD. 2013. http://www.ptsd.va.gov.

30. Zhou X, Wu X, Zhen R. Self-esteem and hope mediate the relations between social support and post-traumatic stress disorder and growth in adolescents following the ya'an earthquake. Anxiety Stress Coping. 2018;31: 32-45.

31. Bovin MJ, Marx BP, Weathers FW, Gallagher MW, Rodriguez P, Schnurr PP, et al. Psychometric properties of the PTSD checklist for diagnostic and statistical manual of mental disorders-fifth edition (PCL-5) in veterans. Psychol Assess. 2016;28:1379-91.

32. Kroenke K, Spitzer RL, Williams JB. The PHQ-9: validity of a brief depression severity measure. J Gen Intern Med. 2001;16:606-13.

33. Wang WZ, Bian Q, Zhao Y, Li X, Wang WW, Du J, et al. Reliability and validity of the Chinese version of the patient health questionnaire (PHQ-9) in the general population. Gen Hosp Psychiatry. 2014;36:539-44.

34. Kocalevent R, Hinz A, Brahler E. 2013. Standardization of the depression screener patient health questionnaire (PHQ-9) in the general population. Gen Hosp Psychiatry. 2013;35:551-5.

35. He XY, Li CB, Qian J, Cui HS, Wu WY. Reliability and validity of ageneralized anxiety disorder scale in general hospital outpatient. Shanghai Arch Psychiatry. 2010;22:200-3.

36. Lowe B, Decker O, Muller S, Brahler E, Schellberg D, Herzog W, et al. Validation and standardization of the generalized anxiety disorder screener (GAD-7) in the general population. Med Care. 2008;46:266-74.

37. Steinberg AM, Brymer MJ, Decker KB, Pynoos RS. The University of California at Los Angeles post-traumatic stress disorder reaction index. Curr Psychiatry Rep. 2004;6:96-100.

38. Connor KM, Davidson JRT. 2003. Development of a new resilience scale: the connor-Davidson resilience scale (CD-RISC). Depression Anxiety. 2003;18:7682

39. Wang L, Shi Z, Zhang Y, Zhang Z. Psychometric properties of the 10-item Connor-Davidson resilience scale in Chinese earthquake victims. Psychiatry Clin Neurosci. 2010;64:499-504.

40. Huang L, Jiang QJ, Ren WH. 1996. Study on the correlation between coping style, social support and psychosomatic symptoms of cancer patients. Chin Ment Health J. 1996;10:160-1.

41. Holmes EA, O'Connor RC, Perry VH, Tracey I, Wessely S, Arseneault L, et al. Multidisciplinary research priorities for the COVID-19 pandemic: a call for action for mental health science. Lancet Psychiatry. 2020;7:547-60.

42. Heneka MT, Golenbock D, Latz E, Morgan D, Brown R. Immediate and longterm consequences of COVID-19 infections for the development of neurological disease. Alzheimers Res Thearpy. 2020;12:69.

43. Dubovsky AN, Arvikar S, Stern TA, Axelrod L. The neuropsychiatric complications of glucocorticoid use: steroid psychosis revisited. Psychosomatics. 2012;53:103-15.

44. Mascolo A, Berrino PM, Gareri P, Castagna A, Capuano A, Manzo C, et al. Neuropsychiatric clinical manifestations in elderly patients treated with hydroxychloroquine: a review article. Inflammopharmacology. 2018;26:11419.

45. Watson D. Differentiating the mood and anxiety disorders: a quadripartite model. Annu Rev Clin Psychol. 2009;5:221-47.

46. Price M, Legrand AC, Brier ZMF, Hebert-Dufresne L. The symptoms at the center: examining the comorbidity of posttraumatic stress disorder, generalized anxiety disorder, and depression with network analysis. J Psychiatr Res. 2019;109:52-8.

47. Wang YY, Hu Z, Feng Y, Wilson A, Chen RS. Changes in network centrality of psychopathology symptoms between the COVID-19 outbreak and after peak. Mol Psychiatry. 2020;25:2140-9.

48. Gavriovic J, Lecic TD, Knezevic G, Priebe S. Predictors of posttraumatic stress in civilians one year after air attacks: a study of Yugoslavian students. J Nerv Ment Dis. 2002:190:257-62.

49. Liu X, Kakade M, Fuller CJ, Fan B, Fang YY, Kong JH, et al. Depression after exposure to stressful events: lessons learned from the severe acute respiratory syndrome epidemic. Compr Psychiatry. 2012;53:15-23.

50. Pappas G, Kiriaze IJ, Giannakis P, Falagas ME. Psychosocial consequences of infectious diseases. Clin Microbiol Infect. 2009;15:743-7.

51. Mulligan K, Jones N, Woodhead C, Davies M, Wessely S, Greenberg N. Mental health of UK military personnel while on deployment in Iraq. $\mathrm{Br} J$ Psychiatry. 2010;197:405-10.

\section{Publisher's Note}

Springer Nature remains neutral with regard to jurisdictional claims in published maps and institutional affiliations. 